\title{
Carotid Intima-Media Thickness in Patients With a History of Kawasaki Disease
}

\author{
Sanne M. Dietz, MD; Carline E. Tacke, MD, PhD; Johan Gort, BSc; \\ Irene M. Kuipers, $\mathrm{MD}, \mathrm{PhD}$; Eric de Groot, $\mathrm{MD}, \mathrm{PhD}$; Albert Wiegman, $\mathrm{MD}, \mathrm{PhD}$; \\ Barbara A. Hutten, PhD; Taco W. Kuijpers, MD, PhD
}

\begin{abstract}
Background: Kawasaki disease (KD) is an acute pediatric vasculitis with coronary artery aneurysms (CAA) as its main complication. Concerns have been raised regarding the possibility of a predisposition of KD to premature cardiovascular disease (CVD) risk later in life. Our aim was to assess carotid intima-media thickness (cIMT), as a surrogate marker of CVD risk, in patients with a history of KD compared with unaffected controls.
\end{abstract}

Methods and Results: B-mode ultrasound cIMT measurements were performed in 168 patients with a history of $\mathrm{KD}$, and 82 controls; 7 patients were excluded because of incomplete cIMT assessments. Mean cIMT ( $\pm S D$ ) was increased in patients with KD compared with controls $(0.378 \pm 0.030 \mathrm{~mm}$ vs. $0.360 \pm 0.027 \mathrm{~mm}$, respectively; $P$ adjusted $<0.0001$ ). If the cIMTs of CAA-negative patients and controls were plotted against age, increased clMT was only apparent at young age. In patients with CAA, increased cIMT was observed over the entire age range.

Conclusions: Our findings show that arterial wall thickening is more apparent in patients with a history of KD as compared with controls. In CAA-negative patients, cIMT is indistinguishable from controls at older age, whereas an increased cIMT is observed at any age in patients with CAA, suggesting a more general and severe effect of KD on the arterial wall. (Circ $J$ 2015; 79: 2682-2687)

\section{Key Words: Cardiovascular disease risk; Carotid intima-media thickness; Coronary artery aneurysm; Kawasaki disease}

$\mathbf{K}$ awasaki disease $(\mathrm{KD})$ is an acute systemic vasculitis that predominantly occurs in children less than 5 years of age. ${ }^{1}$ The disease is thought to be caused by an infectious agent in genetically predisposed children. ${ }^{2}$ Coronary artery aneurysms (CAA) develop in 15-25\% of untreated patients and may lead to myocardial ischemia, infarction, and sudden death. ${ }^{3}$ Although treatment with high-dose intravenous immunoglobulins (IVIG) has reduced this risk to less than $10 \%, \mathrm{KD}$ is the leading cause of acquired heart disease in developed countries. ${ }^{4}$

The disease is self-limiting and only rarely recurs, but there has been ongoing concern that patients both with and without coronary artery involvement may have a predisposition to endothelial damage and premature atherosclerotic disease in adulthood..$^{5-9}$ Thickening of the coronary arterial wall has been shown in persisting and regressed dilatations, but also in always-normal coronary segments. ${ }^{10}$ Since the first case of KD was reported in 1967, patients who have recovered will now be middle-aged or younger, and therefore the follow-up of these patients has not been long enough to establish the natural history of the disease.

To determine if $\mathrm{KD}$ is a risk factor for the future development of cardiovascular disease (CVD), several studies have reported on the carotid intima-media thickness (cIMT) of patients with a history of KD. A thickened intima-media complex can be a result of atherosclerosis, but can also be caused by other processes involving injury and inflammation. As assessed by B-mode ultrasound, it is currently the best-validated non-invasive surrogate marker for cardiovascular risk available. ${ }^{11-13}$ In the past, an increased cIMT has been reported in former $\mathrm{KD}$ patients both with and without CAA, ${ }^{14,15}$ although these findings have not been confirmed by other studies. ${ }^{16-18}$

We hypothesized that, because of their history of a systemic

Received May 21, 2015; revised manuscript received August 17, 2015; accepted August 31, 2015; released online October 6, 2015 Time for primary review: 40 days

Department of Pediatric Hematology, Immunology and Infectious Diseases (S.M.D., C.E.T., T.W.K.), Department of Pediatric Cardiology (I.M.K., A.W.), Emma Children's Hospital, Academic Medical Center, Amsterdam; Department of Vascular Medicine (J.G.), Department of Clinical Epidemiology, Biostatistics and Bioinformatics (B.A.H.), Academic Medical Center, Amsterdam; and Imagelabonline \& Cardiovascular, Eindhoven and Lunteren (E.d.G.), The Netherlands

The first two authors contributed equally (S.M.D., C.E.T.).

Mailing address: Sanne M. Dietz, MD, Department of Pediatric Hematology, Immunology and Infectious Diseases, Emma Children's Hospital, Academic Medical Center, Meibergdreef 9 (H7-270), 1105 AZ Amsterdam, The Netherlands. E-mail: s.m.dietz@amc.uva.nl

ISSN-1346-9843 doi:10.1253/circj.CJ-15-0555

All rights are reserved to the Japanese Circulation Society. For permissions, please e-mail: cj@j-circ.or.jp 
vasculitis, patients with KD have an increased risk of CVD. Therefore, to determine the CVD risk in patients with a history of $\mathrm{KD}$, B-mode ultrasound cIMT measurements (as a surrogate marker of CVD risk) in KD and unaffected control subjects were performed.

\section{Methods}

\section{Participants}

The study was conducted between January 2008 and September 2013 at the Emma Children's Hospital, a tertiary referral center. Children aged 7-20 years with a history of KD were recruited consecutively during follow-up as outpatients. The diagnosis of KD was based on criteria from the American Heart Association. ${ }^{19}$ Patients diagnosed as having KD within 6 months of the study were excluded to minimize the potential confounding influence of (sub)acute inflammation. If multiple IMT measurements had been performed, the last measurement was included in the study.

Unaffected siblings of the children with KD and other unaffected subjects (family from the staff at the hospital) without a history of KD were eligible for the control cohort if they were in the same age range and did not take any cardiovascular medication. All subjects and/or their parents gave informed consent as approved by the institution's Research Ethics Board.

\section{Study Protocol}

A medical (family) history was obtained from all participants, and body height, weight and blood pressure was measured. A non-invasive measurement of the cIMT was performed as described below. The mean arterial pressure (MAP) was calculated using the following formula: (systolic blood pressure $+(2$ times diastolic blood pressure $) / 3$. Using data of the fifth Dutch growth study performed in 2009 in 20,867 children in The Netherlands, standard deviation scores (SDS) for body mass index (BMI) were calculated based on the age and sex of each participant (http://groeiweb.pgdata.nl/calculator.asp).

The medical records of the patients with KD were reviewed retrospectively to collect the following clinical details: age at disease onset, time interval between disease onset and time of study, treatment with IVIG, aspirin and/or steroids, and the presence of CAA. The coronary arteries had been evaluated by $2 \mathrm{D}$ echocardiography. We defined CAA by worst-ever z-scores: CA dimensions as standard deviation units normalized for basal surface area. ${ }^{20,21} \mathrm{We}$ choose to define the CAA by their worst-ever score because even when the lumen of a previously affected coronary artery has returned to its normal size, the artery can still be damaged and thus the initial systemic vasculitis was clearly more severe when compared with children with normal-sized arteries who had never had any enlargement at all. CAA was defined as a coronary z-score $\geq 2.5$, a giant aneurysm was defined as a $\mathrm{z}$-score $\geq 10$ or a diameter $\geq 8 \mathrm{~mm}$. In the patients with $\mathrm{KD}$, a venous blood sample was taken after an overnight fast for measurement of total cholesterol, high-density lipoprotein-cholesterol, low-density lipoprotein (LDL)-cholesterol, triglycerides, apolipoprotein A1, apolipoprotein B, lipoprotein(a) and, apolipoprotein E genotype. LDL-cholesterol was calculated using the Friedewald formula. $^{22}$

\section{Carotid IMT Measurement}

Two experienced and certified ultrasonographers scanned the subjects, using an Acuson Sequioa 512 ultrasound instrument equipped with an 8L5 $8-5 \mathrm{MHz}$ linear array vascular trans- ducer (Siemens AG, Erlangen, Germany). All B-mode ultrasound scans were done according to a standardized protocol. For every subject the right and left common carotid arteries, carotid bulb and internal carotid arterial segments were visualized. One image of each segment was saved as a $2 \times 2 \mathrm{~cm}$ high resolution DICOM still. Image analysis was done off-line in a core lab, for which 20 images were analyzed twice to assess intra-rater reliability. The intraclass correlation coefficient was 0.92 (95\% confidence interval [95\% CI], 0.75-0.97) for the mean cIMT.

One image analyst performed all cIMT measurements blinded for the patient's case status and risk factor levels. The per subject mean combined cIMT was calculated as follows: (mean of the left and right common carotid arteries+the mean of the left and right carotid bulb+the mean of the left and right internal carotid far wall segments)/3. For subjects in whom the scan of one of the segments had failed, the measurement of the same segment of the opposite carotid artery was taken as the mean of both carotid arteries. If both left- and right-side values were unavailable, the IMT was considered missing for that segment, and in that case the mean combined cIMT was also considered missing.

\section{Statistical Analysis}

We evaluated differences in demographics between patients with KD and controls by linear or logistic regression analysis. Differences in cIMT between patients with KD and controls were evaluated using linear regression analyses. We adjusted for the following potential confounders: age, sex, BMI SDS, MAP, family history. In addition, we performed stepwise backward elimination. Furthermore, in the group of KD patients we evaluated if IVIG treatment, IVIG resistance, total cholesterol, LDL-cholesterol and triglycerides were associated with cIMT by linear regression analysis.

An equation for difference in cIMT ( $\triangle \mathrm{IMT})$ was derived by subtracting the equation for patients with KD (if GROUP=1), that is, IMT ${ }^{\mathrm{KD}}=\beta 1 \mathrm{AGE}+\beta 2+\beta 3 \mathrm{AGE}$, from the equation for the unaffected controls (if GROUP $=0$ ), that is, $\mathrm{IMT}^{\mathrm{CO}}=\beta 1 \mathrm{AGE}$. This calculation resulted in $\triangle \mathrm{IMT}=\beta 2+\beta 3 \mathrm{AGE}$. Betas and standard errors were derived from the output of a linear regression analysis for the whole group. Linear and logistic regression analyses were performed using the generalized estimating equation method in the SAS procedure GENMOD to account for correlations within families. The exchangeable correlation structure was used for these models. A P-value $<0.05$ was considered statistically significant. Statistical analyses were performed using SAS release version 9.2 (SAS Institute, Cary, NC, USA) and SPSS version 20.0 software (SPSS Inc, Chicago, IL, USA).

\section{Results}

In total, 168 former patients with KD and 82 controls subjects were enrolled. The control group consisted of 74 unaffected siblings and 8 family members recruited from among the hospital staff; 7 patients were excluded because of missing cIMT segments. Demographic characteristics of the remaining 161 patients with KD and the 82 controls were similar with respect to mean age, sex distribution, mean BMI SDS and MAP (Table 1). Clinical and laboratory data of patients with a history of KD are shown in Table 1 . Median (interquartile range) onset of KD disease was 3.1 (1.2-5.3) years and 145 (90\%) patients were treated with IVIG.

Based on their worst-ever coronary artery z-score, 119 (75\%) had no CAA ( $\mathrm{z}$-score $<2.5$ during the (sub)acute phase) 


\begin{tabular}{|c|c|c|c|}
\hline & Patients $(n=161)$ & Controls $(n=82)$ & $P$ value \\
\hline Age (years) & $12.0 \pm 3.3$ & $12.3 \pm 3.4$ & 0.289 \\
\hline Subgroup 7-10 years & $73(45 \%)$ & $34(41 \%)$ & \\
\hline Subgroup $11-15$ years & $54(34 \%)$ & $30(37 \%)$ & \\
\hline Subgroup 16-20 years & $34(21 \%)$ & $18(22 \%)$ & \\
\hline Male sex, n (\%) & $100(62)$ & $44(54)$ & 0.133 \\
\hline Length $(\mathrm{cm})$ & $154 \pm 0.18$ & $155 \pm 0.17$ & 0.531 \\
\hline Weight (kg) & $46 \pm 16$ & $47 \pm 19$ & 0.338 \\
\hline BMI SD score & $0.47 \pm 1.2$ & $0.26 \pm 1.1$ & 0.185 \\
\hline MAP (mmHg) & $80.1 \pm 7.1$ & $81.4 \pm 8.8$ & 0.242 \\
\hline Family history, $\mathrm{n}(\%)^{\star}$ & $20(12.4)$ & $13(15.9)$ & 0.558 \\
\hline Race & & & 0.755 \\
\hline Caucasian & $131(81.4 \%)$ & $69(84.1 \%)$ & \\
\hline African American & $6(3.7 \%)$ & $0(0 \%)$ & \\
\hline Asian & $3(1.9 \%)$ & $1(1.2 \%)$ & \\
\hline Mixed & $12(7.5 \%)$ & $8(9.8 \%)$ & \\
\hline Other ${ }^{* *}$ & $9(5.6 \%)$ & $4(3.7 \%)$ & \\
\hline Age at disease onset (years) ${ }^{\dagger}$ & $3.1(1.2-5.3)$ & - & - \\
\hline $\begin{array}{l}\text { Interval between disease onset and cIMT } \\
\text { measurement (years) }\end{array}$ & $8.0(6.1-10.9)$ & - & - \\
\hline Treatment with IVIG, n (\%) & $145(90)$ & - & - \\
\hline Lipid profile ${ }^{\dagger \dagger}$ & & \multicolumn{2}{|c|}{ Reference values for age $^{\ddagger}$} \\
\hline Total cholesterol (mmol/L), 7-12 years & $4.17 \pm 0.59$ & \multicolumn{2}{|c|}{$2.8-5.4$} \\
\hline Total cholesterol (mmol/L), 13-20 years & $3.95 \pm 0.72$ & \multicolumn{2}{|c|}{$3.0-5.5$} \\
\hline LDL-cholesterol (mmol/L), 7-12 years & $2.30 \pm 0.54$ & \multicolumn{2}{|c|}{$1.2-3.4$} \\
\hline LDL-cholesterol (mmol/L), 13-20 years & $2.20 \pm 0.60$ & \multicolumn{2}{|c|}{$1.7-3.8$} \\
\hline HDL-cholesterol (mmol/L), 7-12 years & $1.51 \pm 0.40$ & \multicolumn{2}{|c|}{$0.8-1.9$} \\
\hline HDL-cholesterol (mmol/L), 13-20 years & $1.36 \pm 0.29$ & \multicolumn{2}{|c|}{$0.9-1.9$} \\
\hline Triglycerides (mmol/L), 7-12 years ${ }^{\dagger}$ & $0.71(0.53-0.93)$ & \multicolumn{2}{|c|}{$0.3-1.1$} \\
\hline 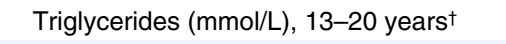 & $0.76(0.50-1.05)$ & \multicolumn{2}{|c|}{$0.4-1.6$} \\
\hline Apolipoprotein E4 genotype, n (\%) & $41(25.5)$ & & \\
\hline
\end{tabular}

Data are expressed as mean \pm standard deviation unless otherwise noted. *First-degree relative with cardiovascular risk factor or disease. ${ }^{* \star}$ Of Turkish, Moroccan or Indo-Surinamese descent. †Median (interquartile range). ${ }^{\dagger}+T o$ convert $\mathrm{mmol} / \mathrm{L}$ to $\mathrm{mg} / \mathrm{dl}$ : for total, high-density lipoprotein and low-density lipoprotein multiply by 38.61 , for triglycerides multiple by 88.50 . ₹Normal Dutch reference values per age category, 2.5-97.5 percentile. ${ }^{31} \mathrm{BMI}$, body mass index; cIMT, carotid intima-media thickness; HDL, high-density lipoprotein; IVIG, intravenous immunoglobulin; KD, Kawasaki disease; LDL, low-density lipoprotein; MAP, mean arterial pressure; SD, standard deviation.

\begin{tabular}{|c|c|c|c|}
\hline & Total (\%) & Mean cIMT $(\mathrm{mm})^{\star \star}$ & $P$ value \\
\hline Controls & $82(100)$ & $0.360 \pm 0.003$ & Reference \\
\hline No enlargement (z-score $<2.5$ ) & $119(73.9)$ & $0.376 \pm 0.002$ & $<0.001$ \\
\hline CAA (z-score 2.5-10) & $31(19.3)$ & $0.373 \pm 0.006$ & 0.064 \\
\hline Giant CAA (z-score $\geq 10$ ) & $11(6.8)$ & $0.412 \pm 0.010$ & $<0.001$ \\
\hline
\end{tabular}

*Based on worst-ever z-score during acute phase of left coronary artery, right coronary artery or left anterior descending artery. ${ }^{\star \star} I M T-m e a n$ adjusted for age and sex (mean \pm standard error). CAA, coronary artery aneurysm. Other abbreviations as in Table 1.

and 42 patients had CAA (31 with a z-score of 2.5-10; and 11 with a $\mathrm{Z}$-score $\geq 10$ or a diameter $\geq 8 \mathrm{~mm})$. Of all patients, 145 $(90 \%)$ had been treated with IVIG and of these, 27 (18.6\%) were IVIG-resistant and had received another dose of IVIG. Baseline characteristics of the CAA-negative and CAA-positive groups are shown in Table $\mathbf{S} 1$.

\section{Carotid IMT}

The mean combined cIMT $( \pm \mathrm{SD})$ was increased in patients with KD when compared with unaffected controls (0.378 $0.030 \mathrm{~mm}$ vs. $0.360 \pm 0.027 \mathrm{~mm} ; \mathrm{P}<0.0001)$. After adjustment for age, sex, BMI, MAP, family history and family relationship, the difference remained statistically significant $(\mathrm{P}<0.0001)$. 


\section{A}

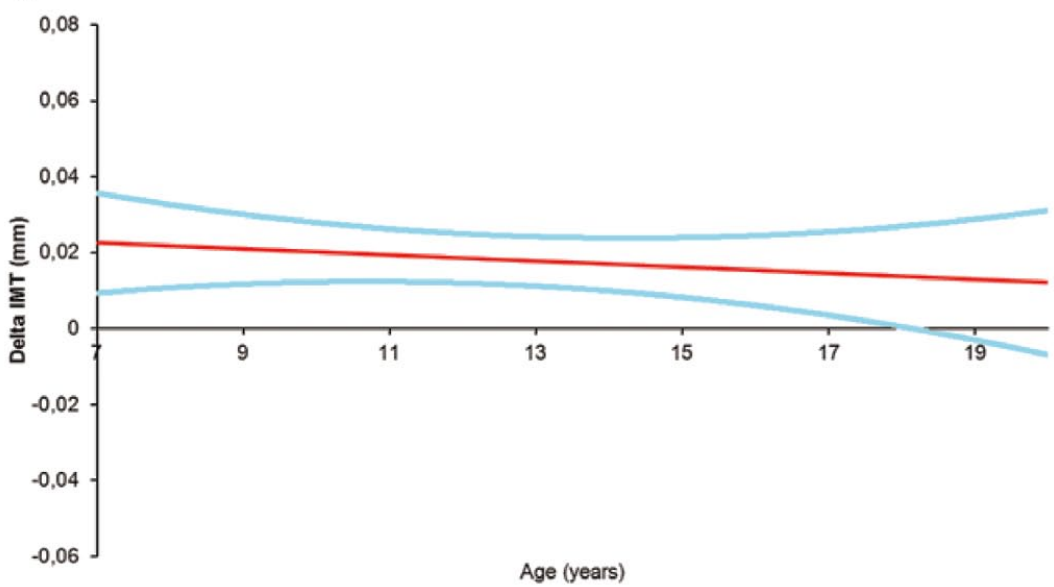

B

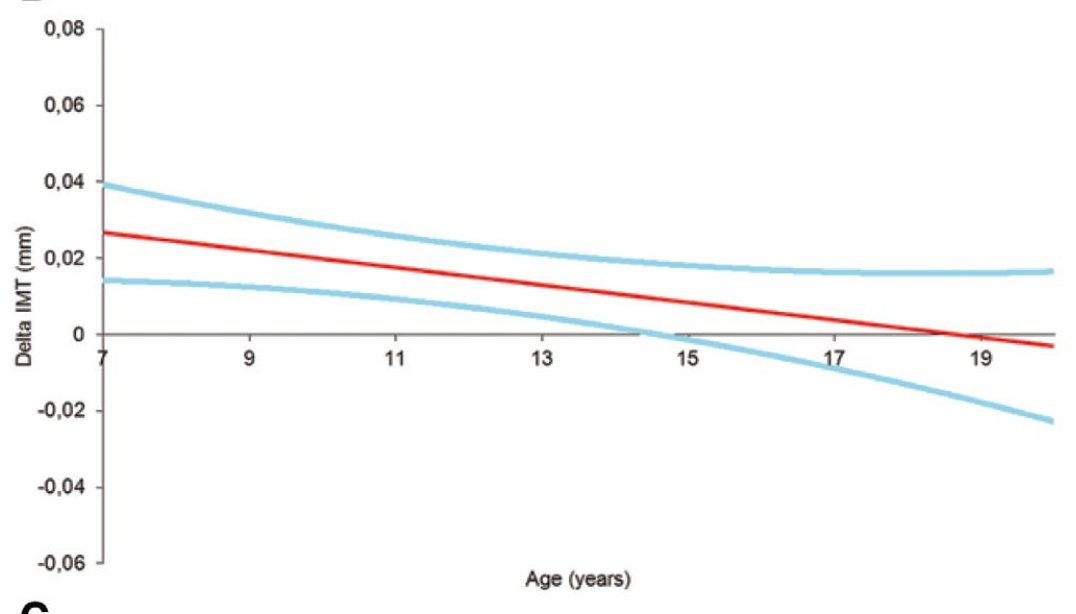

C

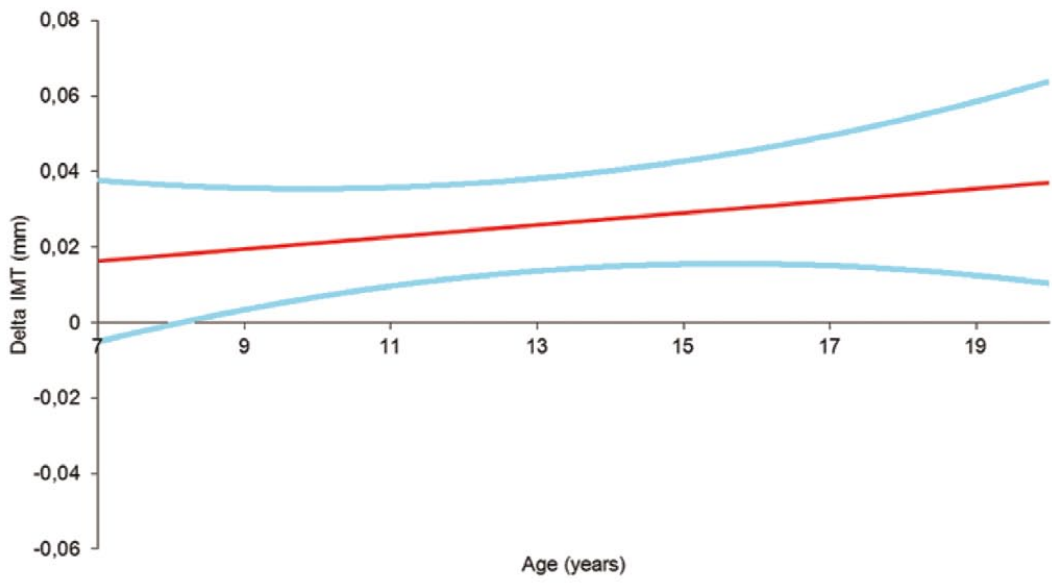

Figure. Difference in mean combined carotid intima-media thickness (cIMT) and 95\% confidence interval $(\mathrm{Cl})$ between patients with a history of Kawasaki disease (KD) and unaffected controls. The difference in mean combined cIMT and $95 \% \mathrm{Cl}$ between patients with a history of $\mathrm{KD}$ and unaffected subjects plotted against age, taking family relationship into account. (A) Difference between the whole group of patients and unaffected subjects $(n=243)$. (B) Difference between the CAA-negative patients and unaffected subjects $(n=201)$. (C) Difference between CAA-positive patients and unaffected subjects $(n=124)$. Mean=thick line; $95 \%$ $\mathrm{Cl}=$ dashed lines. CAA, coronary artery aneurysm.
This result did not change when BMI, MAP and family history were removed using stepwise backward elimination. Table 2 shows the mean cIMT, adjusted for age, sex and family relationship, for the controls and separate subgroups of patients with KD.

In the univariate analysis, IVIG treatment, IVIG resistance, total cholesterol, LDL-cholesterol and triglycerides were not significantly associated with mean cIMT.
For all subjects in the patient group, KD occurred around the age of 3 years. Because the age at cIMT measurement varied considerably (range: 7-20 years), we could explore the association between the difference in cIMT between KD patients and controls $(\triangle \mathrm{IMT})$, and the time since KD onset. For this purpose, we plotted $\triangle \mathrm{IMT}$ against age at cIMT measurement (Figure A).

Based on their worst-ever z-score, plots were also created 
for subgroups without CAA (Figure B) and with CAA (Figure C). In CAA-negative patients, $\triangle \mathrm{IMT}$ diminished with age at the time of cIMT measurement, and disappeared as age increased. In contrast, in patients with $\mathrm{CAA}$ a difference between patients and controls in cIMT remained present at all ages. When we excluded patients with giant aneurysms from the statistical analyses, these results on outcome remained unchanged.

\section{Discussion}

This study shows that children with a history of KD have an increased cIMT compared with unaffected controls. Plotting the difference in cIMT between patients and controls against age indicated that the observed difference in cIMT diminished with increasing age and disappeared in young adulthood in patients without CAA. In contrast, in children with CAA during acute $\mathrm{KD}$ (being either transient or persistent), the difference in cIMT was observed at all ages and remained significantly different from unaffected controls at all ages.

Studies evaluating the cIMT in patients with a history of $\mathrm{KD}$ are limited, and the studies that have been performed mainly included small numbers of patients and have produced conflicting results. We recently published a systematic review and meta-analysis of CVD risk in patients with $\mathrm{KD}$, including a total of 15 cIMT studies. ${ }^{18}$ Some of the studies reported no difference between KD patients and controls, ${ }^{16,17,23,24}$ whereas others found an increased cIMT in CAA-positive patients ${ }^{14,15,25,26}$ or also in CAA-negative patients. ${ }^{14}$ Quality assessment showed that all the studies had some significant methodological limitations. Mean cIMT in the whole KD group and the CAA-positive group did not differ significantly with controls on statistical meta-analysis, although there was a trend toward a thicker cIMT in patients $(0.01 \mathrm{~mm}, 95 \%$ CI $0.00-0.02$ and $0.01 \mathrm{~mm}$, 95\% CI $0.00-0.03 \mathrm{~mm}$, respectively).

In contrast to those findings, the results of the present study showed that subjects with a history of KD had on average a significantly greater mean cIMT compared with unaffected controls. However, the difference in cIMT diminished when analyzed at different ages in the CAA-negative but not in CAA-positive patients. When analyzing the cIMT data in this way (ie, plotted against age at time of measurements), our findings may explain, at least in part, the conflicting data of many of the prior studies that have used different patient groups and variable ages at the time of analysis. Our study suggested that most KD patients tend to 'normalize' over time to eventually fall in the range of normal, unaffected controls. However, our study similarly indicated that the cIMT of CAApositive patients remained abnormal at all ages and could be distinguished as long as we were able to assess cIMT in our cohort of KD patients.

$\mathrm{KD}$ is a vasculitis that predominantly occurs in very young children. ${ }^{27}$ The median age at KD onset in our study cohort was 3.1 years. Therefore, the mean time interval from disease onset to participation in this study increases with age. Although it is unclear which pathophysiologic process results in the increased cIMT of CAA-positive patients, as well as the initially increased cIMT of CAA-negative patients, our findings suggested that this process represents a form of vasculopathy that may be different from premature atherosclerosis. If $\mathrm{KD}$ does result in premature atherosclerosis, $\triangle \mathrm{IMT}$ would be expected to further increase with older age, whereas the cIMT of CAA-negative patients would become indistinguishable from controls in the older children instead.

The assumption that it may be a vasculopathy would be in line with an earlier study looking at the pathology of arteries in KD patients who died of myocardial infarction or heart failure 2-12 years after the onset of KD. ${ }^{28}$ A markedly thickened intima was found in the aneurysms that became stenotic and showed active remodeling of the arterial lesions many years after the disease. This process of remodeling was accompanied by the expression of multiple vascular growth factors, including platelet-derived growth factor and transforming growth factor (TGF)- $\beta 1$, but no fatty streaks or accumulation of macrophages as is seen in (premature) atherosclerosis. Although we may not extrapolate these findings to other vascular structures, including the carotid artery, the finding that TGF- $\beta$ is involved in arterial remodeling could explain the nonprogressive nature of the cIMT in CAA-negative patients up to 15 years after the disease. Such would not be in line with a process of premature atherosclerosis and supports the possibility of a different form of remodeling, provisionally designated as KD-vasculopathy.

In CAA-positive patients this pathway might continue to be active, resulting in a continuing increase of the cIMT. We have previously reported on the presence of vascular growth factors, TGF- $\beta$ and their genetic association with KD. ${ }^{29,30}$ The precise nature of the vasculopathy that might result in a thickened cIMT in KD patients has not yet been determined.

The American Heart Association recommends life-long follow-up for children with and without CAA to assess CVD risk. ${ }^{19}$ Controversy exists on whether there is a need for this in children who never or only transiently experienced CAA. The uncertainty about a possible increased CVD risk based on a systemic vasculitis in the past feeds a big part of this discussion. Our study results indicate that life-long follow-up of children without CAA might not be necessary, although additional factors may be in play. For that reason, longitudinal studies have to be performed to define the real risk for CVD using the same methodology to decide about the follow-up of these children.

A major strength of the present study was the large study group and the consistent use of a standardized carotid imaging and image analysis protocol. Ultrasound measurements of all participants were obtained by 2 experienced sonographers, and 1 image analyst blinded for case or CAA status read all the images.

Some methodological aspects of our study merit discussion. First, we have only included 1 IMT measurement of each patient. The cIMT progression estimates were therefore based on cross-sectional data. Long-term follow-up studies are warranted to assess the 'real' course of cIMT change over time in the same KD patients. Second, patients were stratified based on their 'worst-ever' z-score. Because the study was performed in a tertiary referral center, pediatric cardiologists in centers other than ours generated many of the early echocardiograms. This might have caused misclassification of patients in the stratification for CAA subgroups. Third, although patients were included in a consecutive order at the outpatient clinic, the study population contains a high percentage of patients with CAA, as explained by referral bias with the more severe cases at our tertiary center. This may have overestimated the mean IMT of the total KD group.

\section{Conclusions}

Our study of cIMT in KD patients showed that the signs of early arterial wall changes were more apparent in patients with a history of $\mathrm{KD}$, in particular in those with CAA. When plotting the difference in cIMT between patients and controls in 
patients without CAA, cIMT became indistinguishable from controls with age. In CAA-positive patients an increased cIMT was observed at any age, the latter demonstrating a more severe effect of KD on the arterial wall. Although longitudinal data are missing, this result suggested that follow-up seems justified in CAA-positive patients, but may not be necessary in CAA-negative patients.

\section{Acknowledgments}

This work was supported by the Stinafo Foundation (The Hague, The Netherlands). The sponsor had no role in the study design, the data collection and analysis, the writing of the report, or the decision to submit the manuscript for publication.

\section{References}

1. Kawasaki T, Kosaki F, Okawa S, Shigematsu I, Yanagawa H. A new infantile acute febrile mucocutaneous lymph node syndrome (MLNS) prevailing in Japan. Pediatrics 1974; 54: 271-276.

2. Onouchi Y. Genetics of Kawasaki disease: What we know and don't know. Circ J 2012; 76: 1581-1586.

3. Kato H, Sugimura T, Akagi T, Sato N, Hashino K, Maeno Y, et al. Long-term consequences of Kawasaki disease: A 10- to 21-year follow-up study of 594 patients. Circulation 1996; 94: 1379-1385.

4. Newburger JW, Takahashi M, Burns JC, Beiser AS, Chung KJ, Duffy CE, et al. The treatment of Kawasaki syndrome with intravenous gamma globulin. N Engl J Med 1986; 315: 341-347.

5. Cheung YF, Yung TC, Tam SC, Ho MH, Chau AK. Novel and traditional cardiovascular risk factors in children after Kawasaki disease: Implications for premature atherosclerosis. J Am Coll Cardiol 2004; 43: 120-124.

6. Dhillon R, Clarkson P, Donald AE, Powe AJ, Nash M, Novelli V, et al. Endothelial dysfunction late after Kawasaki disease. Circulation 1996; 94: 2103-2106.

7. Newburger JW, Burns JC, Beiser AS, Loscalzo J. Altered lipid profile after Kawasaki syndrome. Circulation 1991; 84: 625-631.

8. Silva AA, Maeno Y, Hashmi A, Smallhorn JF, Silverman ED, McCrindle BW. Cardiovascular risk factors after Kawasaki disease: A case-control study. J Pediatr 2001; 138: 400-405.

9. JCS Joint Working Group. Guidelines for diagnosis and management of cardiovascular sequelae in Kawasaki disease (JCS 2013): Digest version. Circ J 2014; 78: 2521-2562.

10. Suzuki A, Yamagishi M, Kimura K, Sugiyama H, Arakaki Y, Kamiya T, et al. Functional behavior and morphology of the coronary artery wall in patients with Kawasaki disease assessed by intravascular ultrasound. J Am Coll Cardiol 1996; 27: 291-296.

11. de Groot E, Hovingh GK, Wiegman A, Duriez P, Smit AJ, Fruchart JC, et al. Measurement of arterial wall thickness as a surrogate marker for atherosclerosis. Circulation 2004; 109: III33-III38.

12. Touboul PJ, Hennerici MG, Meairs S, Adams H, Amarenco P, Bornstein N, et al. Mannheim carotid intima-media thickness and plaque consensus (2004-2006-2011): An update on behalf of the advisory board of the 3rd, 4th and 5th watching the risk symposia, at the 13th, 15th and 20th European Stroke Conferences, Mannheim, Germany, 2004, Brussels, Belgium, 2006, and Hamburg, Germany, 2011. Cerebrovasc Dis 2012; 34: 290-296.

13. Lorenz MW, Markus HS, Bots ML, Rosvall M, Sitzer M. Prediction of clinical cardiovascular events with carotid intima-media thickness: A systematic review and meta-analysis. Circulation 2007; 115: 459-467.

14. Cheung YF, Wong SJ, Ho MH. Relationship between carotid intimamedia thickness and arterial stiffness in children after Kawasaki disease. Arch Dis Child 2007; 92: 43-47.

15. Noto N, Okada T, Yamasuge M, Taniguchi K, Karasawa K, Ayusawa M, et al. Noninvasive assessment of the early progression of atherosclerosis in adolescents with Kawasaki disease and coronary artery lesions. Pediatrics 2001; 107: 1095-1099.

16. Ikemoto Y, Ogino H, Teraguchi M, Kobayashi Y. Evaluation of preclinical atherosclerosis by flow-mediated dilatation of the brachial artery and carotid artery analysis in patients with a history of Kawasaki disease. Pediatr Cardiol 2005; 26: 782-786.

17. Selamet Tierney ES, Gal D, Gauvreau K, Baker AL, Trevey S, O'Neill SR, et al. Vascular health in Kawasaki disease. J Am Coll Cardiol 2013; 62: 1114-1121.

18. Dietz SM, Tacke CE, Hutten BA, Kuijpers TW. Peripheral endothelial (dys)function, arterial stiffness and carotid intima-media thickness in patients after Kawasaki Disease: A systematic review and meta-analyses. PloS One 2015; 10: e0130913, doi:10.1371/journal. pone.0130913.

19. Newburger JW, Takahashi M, Gerber MA, Gewitz MH, Tani LY, Burns JC, et al. Diagnosis, treatment, and long-term management of Kawasaki disease: A statement for health professionals from the Committee on Rheumatic Fever, Endocarditis and Kawasaki Disease, Council on Cardiovascular Disease in the Young, American Heart Association. Circulation 2004; 110: 2747-2771.

20. McCrindle BW, Li JS, Minich LL, Colan SD, Atz AM, Takahashi $\mathrm{M}$, et al. Coronary artery involvement in children with Kawasaki disease: Risk factors from analysis of serial normalized measurements. Circulation 2007; 116: 174-179.

21. Manlhiot C, Millar K, Golding F, McCrindle BW. Improved classification of coronary artery abnormalities based only on coronary artery z-scores after Kawasaki disease. Pediatr Cardiol 2010; 31: 242-249.

22. Friedewald WT, Levy RI, Fredrickson DS. Estimation of the concentration of low-density lipoprotein cholesterol in plasma, without use of the preparative ultracentrifuge. Clin Chem 1972; 18: 499-502.

23. Ishikawa T, Iwashima S. Endothelial dysfunction in children within 5 years after onset of Kawasaki disease. J Pediatr 2013; 163: $1117-1121$.

24. Oguri M, Nakamura T, Tamanuki K, Akita C, Kitaoka C, Saikawa $\mathrm{Y}$, et al. Subclinical arterial stiffness in young children after Kawasaki disease. Cardiol Young 2014; 24: 87-94.

25. Cheung YF, O K, Woo CW, Armstrong S, Siow YL, Chow PC, et al. Oxidative stress in children late after Kawasaki disease: Relationship with carotid atherosclerosis and stiffness. BMC Pediatr 2008; 8: 20 .

26. Noto N, Okada T, Abe Y, Miyashita M, Kanamaru H, Karasawa K, et al. Characteristics of earlier atherosclerotic involvement in adolescent patients with Kawasaki disease and coronary artery lesions: Significance of gray scale median on B-mode ultrasound. Atherosclerosis 2012; 222: 106-109.

27. Tacke CE, Breunis WB, Pereira RR, Breur JM, Kuipers IM, Kuijpers TW. Five years of Kawasaki disease in the Netherlands: A national surveillance study. Pediatr Infect Dis J 2014; 33: 793-797.

28. Suzuki A, Miyagawa-Tomita S, Komatsu K, Nishikawa T, Sakomura Y, Horie T, et al. Active remodeling of the coronary arterial lesions in the late phase of Kawasaki disease: Immunohistochemical study. Circulation 2000; 101: 2935-2941.

29. Breunis WB, Davila S, Shimizu C, Oharaseki T, Takahashi K, van Houdt M, et al. Disruption of vascular homeostasis in patients with Kawasaki disease: Involvement of vascular endothelial growth factor and angiopoietins. Arthritis Rheum 2012; 64: 306-315.

30. Shimizu C, Jain S, Davila S, Hibberd ML, Lin KO, Molkara D, et al. Transforming growth factor-beta signaling pathway in patients with Kawasaki disease. Circ Cardiovasc Genet 2011; 4: 16-25.

31. de Rijke YBL, Lindemans J, Boot AM. Referentiewaarden klinische chemia en endocrinicologie. In: Derksen-Lubsen G, Moll HA, Oudesluys-Murphy HM, Sprij AJ, editors. Compendium kindergeneeskunde. Houten: Bohn Stafleu van Loghum, 2011; 653-666 (in Dutch).

\section{Supplementary Files}

Supplementary File 1

Table S1. Baseline characteristics of CAA-positive and CAA-negative subgroups of KD patients according to worst-ever z-score

Please find supplementary file(s);

http://dx.doi.org/10.1253/circj.CJ-15-0555 